\title{
Evitación experiencial y uso abusivo del smartphone: un enfoque bayesiano
}

\section{Experiential avoidance and excessive smartphone use: a Bayesian approach}

\author{
Ana María Ruiz-Ruano*, María Dolores López-Salmerón**, Jorge L. Puga*** \\ * UCAM Universidad Católica San Antonio de Murcia, Facultad de Ciencias Jurídicas y de la Empresa. \\ ** Programa de Doctorado en Ciencias de la Salud. Universidad Católica de Murcia (UCAM). \\ *** UCAM Universidad Católica San Antonio de Murcia, Facultad de Ciencias de la Salud.
}

\section{Resumen}

El uso del teléfono móvil se ha convertido en una actividad cotidiana en nuestro entorno más cercano. Dicho uso, según investigaciones recientes, tiene tanto aspectos positivos como negativos. Aunque hay controversia en cuanto a la denominación del fenómeno, se aprecia cierta preocupación por las consecuencias negativas que tiene el uso excesivo del teléfono móvil. Este estudio analiza la relación que se establece entre el uso abusivo del teléfono móvil y la evitación experiencial. Se utilizó una muestra compuesta por 1176 participantes (828 fueron mujeres) con edades comprendidas entre los 16 y los 82 años $(M=30.97 ; D T=12.05)$. Se empleó la escala SAS-SV para valorar el uso problemático del móvil y el AAQ-II para la evitación experiencial. Para modelar la relación que se establece entre las variables se hizo uso de inferencia bayesiana y redes bayesianas. Los resultados muestran una relación directa entre el uso abusivo, la evitación experiencial y las redes sociales. Además, los datos sugieren que el sexo juega un papel mediador entre estas variables. Estos resultados son útiles para entender el uso saludable y patológico del teléfono móvil así como para orientar el tratamiento de los trastornos que pueden surgir de un mal uso de estos dispositivos.

Palabras clave: Evitación experiencial; Smartphone; Adicción; Redes sociales; Inferencia bayesiana.

\begin{abstract}
The smartphone is a common tool in our everyday lives. However, recent research suggests that using the smartphone has both positive and negative consequences. Although there is no agreement on the concept or the term to label it, researchers and clinical practitioners are worried about the negative consequences derived from excessive smartphone usage. This study aims to analyse the relationship between smartphone addiction and experiential avoidance. A sample of 1176 participants (828 women) with ages ranging from 16 to 82 ( $M$ $=30.97 ; S D=12.05$ ) was used. The SAS-SV scale was used to measure smartphone addiction and the AAQ-II to assess experiential avoidance. To model the relationship between variables, Bayesian inference and Bayesian networks were used. The results show that experiential avoidance and social networks usage are directly related to smartphone addiction. Additionally, the data suggests that sex is playing a mediating role in the observed relationship between these variables. These results are useful for understanding healthy and pathological interaction with smartphones and could be helpful in orienting or planning future psychological interventions to treat smartphone addiction.
\end{abstract}

Keywords: Experiential avoidance; Smartphone; Addiction; Social networks; Bayesian inference.

Recibido: Marzo 2018; Aceptado: Septiembre 2018.

Enviar correspondencia a:

Ana María Ruiz-Ruano García, Campus de los Jerónimos s/n, 30107, Guadalupe, Murcia.

amruiz@ucam.edu. 
$\mathrm{E}$ 1 uso del teléfono móvil en la sociedad actual ha pasado de ser un fenómeno aislado a convertirse en una actividad casi imprescindible en nuestras vidas (Odgers, 2018). Así, autores como Buchinger, Kriglstein, Brandt y Hlavacs (2011) califican el teléfono móvil como un instrumento indispensable para la actual vida social y laboral. Los teléfonos móviles, o los denominados smartphones, presentan una serie de ventajas como, por ejemplo, permitirnos estar más comunicados, promover un aumento de la identidad grupal o ser una vía fácil para la transmisión de las emociones (Tresáncoras, García-Oliva y Piqueras, 2017). También tiene efectos en los niveles de autonomía, prestigio social, es una fuente para el ocio y se desdibuja como una vía para el fomento y establecimiento de relaciones sociales (Chóliz, Villanueva y Chóliz, 2009). Por otro lado, presentan aspectos positivos como herramientas para la intervención en ciertas patologías y la recogida de información mediante aplicaciones centradas en la salud (p.e., Capon, Hall, Fry y Carter, 2016; Gustafson et al., 2014; Kuhn et al., 2017; Seoane y Álvarez, 2012). No obstante, y pese a los aspectos positivos de su uso (Odgers, 2018), encontramos un amplio abanico de estudios en la literatura científica que señala posibles efectos adversos que pueden derivarse de su mal uso.

Sin embargo, no existe consenso en la manera de denominar el efecto pernicioso que tiene el móvil en la salud (Carbonell, Fúster, Chamorro y Oberst, 2012; Simó, Martínez, Ballester y Domínguez, 2017). Algunos autores abogan por la terminología de uso abusivo (p.e., Chóliz et al., 2009), uso problemático (p.e., Marín, Carballo y Coloma-Carmona, 2018; Pedrero-Pérez et al., 2018; Simó et al., 2017; Tresáncoras et al., 2017), uso desadaptativo (p.e., Gil, del Valle, Oberst y Chamarro, 2015) o incluso adicción (p.e., Carbonell et al., 2012). En cualquier caso, parece que la mayoría de los estudios tratan de situar este fenómeno dentro de las denominadas adicciones comportamentales. Sin embargo, hablar de adicción al móvil, tal como están recogidas las adicciones dentro de los manuales de diagnóstico, y sobre todo si tomamos como referencia el actual DMS-5 (American Pychiatric Association [APA], 2013), podría ser legítimo a la vez que cuestionable dada la falta de consenso que existe al respecto (Simó et al., 2017).

En cualquier caso, existen ciertos estudios que hacen énfasis en las consecuencias perjudiciales de este uso abusivo o problemático del móvil, señalando desde consecuencias a nivel físico y psicológico (estrés o ansiedad) como a nivel social, familiar, escolar o laboral (p.e., Babín, 2009; Echeburúa y Corral, 2010; Hardell, Carlbert y Hansson, 2011; Klawer et al., 2014; Lee, Kang y Shin, 2015; Marín et al., 2018; Zarghami, Khalilian, Setareh y Salehpour, 2015). Dentro de estas consecuencias negativas, también se ha descrito el surgimiento de nuevos comportamientos desadaptativos vinculados a su uso (véase, por ejemplo, Bragazzi y Puente, 2014; Gil et al., 2015; Karadağ et al., 2015; Krasno- va, Abramova, Notter y Baumann, 2016; McDaniel y Coyne, 2016; Mendoza y Cuñarro, 2016; Roberts y David, 2016; Rodríguez, 2015; Wang, Xie, Wang, Wang y Lei, 2017).

Desde un punto de vista teórico, parece que las mujeres, por su tendencia al mantenimiento en mayor medida que los hombres de comportamientos prosociales, tienden a ser más proclives a un uso problemático del móvil (Veissière y Stendel, 2018). Karadağ y colaboradores (2015), en la misma línea, señalan que el hecho de que las mujeres hagan un mayor uso de los móviles que los hombres es debido a su deseo por gustar y por querer compartir sus vidas. Por su parte, existen una serie de estudios en los que se señala que las mujeres dedican más tiempo al uso del móvil, a la mensajería instantánea y a las redes sociales (p.e., Chóliz, 2012; Chóliz et al., 2009, De-Sola, Rodríguez y Rubio, 2016; Gil et al., 2015; Pedrero-Pérez et al., 2018; Tresáncoras et al., 2017). Otro aspecto relevante recogido por algunos de estos estudios es que, en algunos casos, las mujeres señalan que el uso del móvil les ayuda a enfrentarse a estados de ánimo displacenteros (Chóliz et al., 2009; De-Sola et al., 2016) e incluso para "superar el aburrimiento, manejar la ansiedad, o en momentos en que se encuentran tristes o solas" (Chóliz et al., 2009, p.84). Por último, en una revisión realizada por Carbonell et al. (2012) sobre estudios españoles, se señala que las mujeres presentan más problemas con el uso de los móviles y además consideran su uso más problemático que en el caso de los hombres.

Además, el teléfono móvil es una de las herramientas más frecuentes para acceder a las redes sociales, si no la más empleada. Algunos estudios muestran que las redes sociales permiten desarrollar aspectos positivos en las personas (p.e., Pedrero, Rodríguez y Ruiz, 2012) y han transformado la forma de establecer relaciones sociales (p.e., Echeburúa y Corral, 2010; Orozco, 2015). Sin embargo, pese a las claras ventajas que proporcionan este tipo de tecnologías, existen aspectos negativos relacionados con su uso. Por ejemplo, se considera que el uso de las redes sociales es un factor de riesgo para el uso abusivo del teléfono móvil (Deursen, Bolle, Hegner y Kommers, 2015; Griffiths, 2000; Zhitomirsky-Geffet y Blau, 2016), para problemas de salud mental o estrés (Pedrero-Pérez et al., 2018) y sobre todo para la población adolescente (Arab y Díaz, 2015; Chóliz et al., 2009; Tresáncoras et al., 2017).

Desde un punto de vista psicológico, una posible explicación del uso desadaptativo del móvil en el ámbito de las redes sociales podría estar vinculada con la tendencia a huir de los sentimientos aversivos que nos provoca la realidad no-virtual, sobre todo en el caso de las mujeres como se ha comentado anteriormente (Carbonell et al., 2012; Chóliz et al., 2009). El concepto de evitación experiencial o trastorno de evitación experiencial se acuñó, precisamente, para hacer alusión a esa tendencia evitativa desadaptativa que se relaciona con diferentes trastornos mentales (Hayes, Wilson, Gifford, Follete y Stroashal, 1996). Bajo este paradigma 
se entiende que ciertos trastornos psicológicos son el resultado de un patrón persistente de evitación desadaptativo orientado hacia los eventos internos negativos que produce malestar de forma crónica y generalizada. Se entiende que este patrón de funcionamiento disfuncional está basado en procesos de regulación verbal (p.e., Hayes, Brownstein, Zettle, Rosenfard y Korn, 1986; Hayes, Strosahl y Wilson, 1999; Hayes, Zettle y Rosenfarb, 1989; Wulfert, Greenway, Farkas, Hayes y Dougher, 1994) y, como consecuencia, la persona experimenta una limitación social, personal y/o laboral que se transfiere a diferentes contextos vitales implicando un coste personal elevado (Wilson y Luciano, 2012).

En el contexto de las adicciones se ha observado que el uso inapropiado o abusivo de sustancias químicas como el tabaco o el alcohol está relacionado con la evitación experiencial. Por ejemplo, en 2016, Levin et al. reportaron que las personas que consumían alcohol abusivamente mostraban mayores puntuaciones en evitación experiencial. Garey, Farris, Schmidt y Zvolensky (2016) sugieren que el hábito tabáquico podría explicarse por un mecanismo de evitación experiencial condicionado por los estresores habituales de la vida cotidiana. En este mismo sentido, Watson, Heffner, McClure y Bricker (2017) aportan evidencias que sugieren que los fumadores con altos niveles de ansiedad social también presentan una mayor evitación experiencial. Bahrami y Asghari (2017), por su parte, observaron que la evitación experiencial y estilos de afrontamiento inapropiados podrían explicar el fracaso terapéutico con pacientes dependientes de la metanfetamina. Además, concluyeron que el uso de la Terapia de Aceptación y Compromiso como técnica orientada a la reducción de la evitación experiencial (Hayes et al., 1999) optimizaba las perspectivas de mejora en estos pacientes. Por su parte, Buckner y Zvolensky (2014) también obtuvieron evidencias de que un patrón evitativo condicionaba la ansiedad social que mostraban los consumidores de cannabis.

Existen menos estudios que relacionen la evitación experiencial con adicciones comportamentales. De hecho, el único trastorno vinculado más directamente con la idea de adicción es el juego patológico y se señala (criterio A.5 del DSM-5) que el comportamiento problemático aparece como consecuencia de sensaciones desagradables como el desasosiego, el desamparo, la depresión o la ansiedad (APA, 2013). Por otro lado, el trastorno de juego por internet está recogido en el DSM-5 dentro de las afecciones que necesitan más estudio y si en el futuro fuese reconocido como trastorno per se, nos encontraríamos ante el primer trastorno derivado del uso de las tecnologías. Según la APA (2013), uno de los criterios diagnósticos del trastorno de juego por internet está directamente relacionado con la evitación experiencial ya que se indica (criterio 8) que el comportamiento patológico aparece para "evadirse o aliviar un afecto negativo" (p.795). En el estudio reciente de García-Oliva y Piqueras (2016) se señala que existe relación entre la evitación experiencial y el uso de tecnologías de la información y la comunicación (TICs). Concretamente, indican que las TICs son utilizadas como vía para escapar de estímulos internos aversivos.

Por consiguiente, dado que la evitación experiencial está relacionada con algunos trastornos adictivos (p.e., Hayes et al., 1996), también podría esperarse una relación de esta variable con el uso abusivo del móvil. Dado que, como se ha indicado más arriba, las redes sociales juegan un papel muy relevante en el uso de los dispositivos móviles, cabría esperar que el uso de estas herramientas de interacción social pudiese ser explicada por altos niveles de evitación experiencial. Por otra parte, también cabría esperar que el uso problemático del móvil estuviese relacionado con el hecho de ser hombre o mujer dado que algunos autores señalan que esta variable se relaciona con el uso desadaptativo del móvil (p.e., Chóliz, 2012; Chóliz et al., 2009; Gil et al., 2015; Pedrero-Pérez et al., 2018; Tresáncoras et al., 2017). Para estudiar estas relaciones hipotetizadas entre el uso abusivo del móvil, la preferencia por aplicaciones de redes sociales y la evitación experiencial, utilizaremos algoritmos de aprendizaje estructural automático de redes bayesianas (p.e., Nagarajan, Scutari y Lèbre, 2013, Ruiz-Ruano, 2015; Scutari, 2010). Las redes bayesianas son herramientas estadísticas multivariadas que permiten modelar gráficamente las relaciones probabilísticas que se establecen entre un conjunto de variables (Cowe1l, Dawid, Lauritzen y Spiegelhalter, 1999; Edwards, 1998; Puga, Krzywinski y Altman, 2015). Pese a su utilidad potencial, el aprendizaje estructural automático de redes bayesianas ha sido relativamente poco utilizado en psicología en comparación con otras aplicaciones que se dan a este tipo de herramientas (p.e., López, García, De la Fuente y De la Fuente, 2007; Ruiz-Ruano, 2015). Si los datos apuntasen en la dirección de las hipótesis planteadas, nuestro trabajo podría ser útil desde el punto de vista clínico o aplicado a la hora de planificar intervenciones destinadas a prevenir o solventar problemas relacionados con el uso abusivo del teléfono móvil.

\section{Método}

\section{Participantes}

La muestra no probabilística seleccionada por medio de un muestreo tipo bola de nieve estuvo compuesta por un total de 1176 participantes, de los cuales 348 fueron hombres $(29.6 \%)$ y el resto mujeres (828). Sus edades estuvieron comprendidas entre 16 y $82(M=30.97 ; D T=12.05)$. El $59.7 \%$ de los participantes indicaron tener pareja (38.4\%) o estar casados $(21.3 \%)$, seguidos por aquellos que indicaron estar solteros $(36.3 \%)$, divorciados $(2.5 \%)$, viudos $(0.3 \%)$, y un $1.1 \%$ que indicaron tener un estado civil diferente de los anteriores. Con respecto al nivel educativo, la mayor parte de la muestra señaló estar en posesión de algún título 
universitario (64.8\%), el $0.4 \%$ indicó no tener estudios, y el resto de los participantes indicaron estar en posesión del título de bachillerato, formación profesional, ESO o estudios primarios. En cuanto al estatus profesional, el $44.5 \%$ de los participantes indicaron ser trabajadores, el $37.8 \%$ estudiantes, el $9.9 \%$ indicó encontrarse en situación de desempleo, el $2.4 \%$ jubilados, y, por último, el $2 \%$ indicaron ser amas de casa.

\section{Instrumentos}

Se elaboró un cuestionario con la plataforma Google Forms $^{\circledR}$ en el que se recogió información sociodemográfica (edad, sexo, estado civil, nivel de estudios y estatus laboral) e información relacionada con el smartphone (aplicación más utilizada, tiempo de uso, razones de uso y número de teléfonos móviles). El cuestionario incluyó una pregunta sobre la aplicación que con más preferencia se usaba con el dispositivo móvil, cuyas respuestas fueron recodificadas para representar la preferencia de los participantes por las redes sociales frente al resto de aplicaciones disponibles en sus teléfonos móviles. Además, se incluyó una escala para valorar el nivel de adicción al smartphone, y un cuestionario para evaluar el nivel de evitación experiencial.

La escala de adicción al smartphone utilizada (SAS-SV) es la versión corta de la escala de adicción al smartphone (SAS) creada originalmente por Kwon et al. (2013a). Esta versión reducida diseñada por Kwon, Kim, Cho y Yang (2013b) cuenta con una consistencia interna $\alpha=.91$. En este estudio se ha utilizado la adaptación al español realizada por López-Fernández (2015) que obtuvo un $\alpha$ de Cronbach de .88 en el correspondiente estudio de adaptación. La escala está formada por 10 ítems basados en la dependencia de sustancias y el trastorno de juego patológico propuesto por el DSM-IV (APA, 1994, 2000). El formato de respuesta se presenta en una escala tipo Likert de 6 puntos, donde 1 se corresponde con "Totalmente en desacuerdo" y 6 con "Totalmente de acuerdo". La puntuación de la escala oscila entre 10 y 60, existiendo un mayor riesgo de adicción al smartphone a medida que la puntuación es más elevada. Los índices de consistencia interna obtenidos en este estudio para la escala SAS-SV son: $\alpha=.87$, IC95\%: .86, .89, y $\omega=.88$.

Para medir la evitación experiencial, o inflexibilidad cognitiva, se utilizó la versión del Cuestionario de Aceptación y Acción (Acceptance and Action Questionnaire II o AAQII) presentada por Ruiz, Langer, Luciano, Cangas y Beltrán (2013). La primera versión de este test fue desarrollada por Hayes et al. (2000) y Hayes et al. (2004) con base en diferentes experiencias clínicas y obteniéndose un alfa de consistencia interna de .7. Bond et al. (2011) desarrollaron la segunda versión del test que mostraba mayores niveles de consistencia interna $(\alpha=.97)$ y que contenía un menor número de ítems. La escala está formada por siete ítems con respuestas tipo Likert de siete puntos que expresan el grado de veracidad que el participante atribuye a cada ítem según su experiencia. En esta aplicación del test, los valores observados de consistencia interna son: $\alpha=.89$, IC95\%: .89, $.90, \mathrm{y} \omega=.90$.

\section{Procedimiento}

El formulario electrónico se difundió por medio de la aplicación de mensajería instantánea WhatsApp ${ }^{\circledR}$, utilizando las redes sociales $\left(\right.$ Facebook $^{\circledR}$ y Twitter $^{\circledR}$ ) y por medio de correo electrónico. Para el comienzo en la recogida de datos se contó con la colaboración de estudiantes universitarios que cumplimentaron el formulario y lo difundieron entre sus contactos de las redes sociales. $\mathrm{Al}$ inicio del cuestionario se detallaban los objetivos del estudio y se indicaba el anonimato y confidencialidad de los datos recogidos con el mismo. Así mismo, se instaba al participante a difundir el formulario entre sus contactos de las redes sociales. La difusión y recogida de datos dio comienzo el 24 de noviembre de 2016 y se dio por finalizada el 30 de enero de 2017.

\section{Análisis de datos}

La estrategia analítica aplicada está en consonancia con la propuesta de Cohen, Cohen, West y Aiken (2003) considerando los modelos correlacionales como marco general para el estudio del comportamiento. Así, por ejemplo, las correlaciones entre las variables cuantitativas y las dicotómicas (como, por ejemplo, el hecho de disponer de más de un teléfono móvil o no) fueron estimadas como coeficientes estandarizados de los correspondientes modelos de regresión lineal que explicarían la variable cuantitativa en función de la pertenencia grupal en la variable dicotómica. Para obtener la matriz de correlaciones entre las variables del estudio y los factores de Bayes favorables a la hipótesis alternativa frente a la nula $\left(F B_{10}\right)$ se utilizó la versión 0.9 del programa estadístico JASP (JASP Team, 2018).

El factor de Bayes así calculado expresa cuanto más verosímil o probable es la hipótesis alternativa frente a la hipótesis nula (Kass y Raftery, 1995). Un factor de Bayes igual a uno indicaría que la hipótesis alternativa es igual de probable que la hipótesis nula teniendo en cuenta los datos observados. Por su parte, un factor de Bayes mayor que uno indicaría cuan más probable es la hipótesis alternativa frente a la hipótesis nula. Así, por ejemplo, un $F B_{10}$ igual a dos indicaría que la hipótesis alternativa es dos veces más probable que la hipótesis nula, mientras que un $F B_{10}$ igual a 100 indicaría que la hipótesis alternativa es 100 veces más probable que la hipótesis nula. Para estimar los Factores de Bayes se utilizó la distribución Cauchy por defecto $(r=1)$ sugerida por Rouder, Speckman, Sun y Morey (2009). Esta distribución previa ha mostrado ser una opción equilibrada en términos de los elementos clave implícitos en la toma de decisiones estadística según los estudios de simulación realizados hasta el momento (Jeon y De Boeck, 2017). 
Por su parte, los modelos estructurales de red bayesiana fueron estimados utilizando el paquete «bnlearn» (Scutari, 2010) para R en su versión 4.2. Se utilizaron seis algoritmos diferentes para encontrar el modelo que mejor se ajustase a los datos. Dos de los algoritmos utilizados utilizaban métodos de constricción de modelos (Grow-Shrink e Incremental Association), dos estuvieron basados en el ajuste (Hill-Climbing y tabu search), mientras que los dos restantes fueron mixtos (Max-min Hill-Cimbing y Restricted Maximization). Para estudiar la bondad de ajuste de los modelos estimados por cada algoritmo se llevaron a cabo dos métodos diferentes. En primer lugar, se dividió la muestra en un conjunto de estimación que contuvo el $70 \%$ de las observaciones y el $30 \%$ (subconjunto de test) restante se utilizó para valorar el grado en que los datos se ajustaban a los modelos estimados en la fase de estimación. Para valorar la bondad de ajuste de la estimación se utilizó el logaritmo de la verosimilitud, el Criterio de Información Akaike (AIC), y el Criterio Bayesiano de Información (BIC) (Scutari y Denis, 2014). Se considera que cuanto mayores son estos valores, mejor es el ajuste del modelo a los datos. El segundo procedimiento de validación consistió en dividir aleatoriamente en partes iguales el conjunto de datos 2000 veces para estimar la compensación que se produce en el logaritmo de la verosimilitud de una a otra estimación (Koller y Friedman, 2010). En este caso, menores valores de compensación en el logaritmo de la verosimilitud se interpretarían como un mejor ajuste. Por último, para estimar la fuerza de asociación de cada enlace de la red bayesiana se analizó el cambio que se produce en la verosimilitud, en el AIC y en el BIC al eliminar la correspondiente arista del modelo (Scutari y Denis, 2014). En este caso, cuanto más pequeños son los valores de la verosimilitud, del AIC o del BIC cuando un enlace es eliminado del modelo, más relevante o influyente se considera ese enlace para la red testada. Es decir, en este caso los estadísticos de bondad de ajuste valoran el grado en que empeora el modelo cuando un enlace es eliminado del mismo. Así, cuanto más pequeño es el valor del estadístico más se supone que empeora el modelo si eliminásemos este enlace.

\section{Resultados}

Como se puede apreciar en la Tabla 1, las variables que se asocian con más intensidad y en sentido positivo son el número de horas que se utiliza el teléfono móvil y el tiempo que la persona invierte en la aplicación preferida. La segunda correlación positiva más importante que se observa atendiendo a su magnitud es la que se da entre la puntuación de evitación experiencial medida con la AAQ-II y la puntuación de adicción al teléfono móvil estimada con la escala SAS-SV. La evitación experiencial también correlaciona positiva y significativamente con el tiempo que los usuarios invierten en su aplicación preferida y las horas que invierten en el teléfono móvil. También se observa que, con la misma intensidad de asociación y en sentido positivo, existe relación entre la puntuación de adicción medida con la escala SAS-SV y las horas de uso del teléfono móvil, así como con el tiempo invertido en la aplicación preferida. Como se puede observar en la Tabla 1, los factores de Bayes estimados para estas correlaciones apuntan a que los datos observados podrían considerarse como evidencias decisivas $\left(F B_{10}>100\right)$ en favor de que las correlaciones entre las susodichas variables son genuinamente diferentes de cero (Jeffreys, 1948). Dicho de otro modo, atendiendo a los Factores de Bayes asociados a estas correlaciones podríamos decir que la hipótesis de correlación genuina entre estas variables es, como mínimo, 600 millones de veces más probable (Factor de Bayes asociado a la correlación observada entre evitación experiencial y horas que se dedican al uso del teléfono móvil) que la hipótesis de no correlación.

Los resultados muestran (Tabla 1) que las horas invertidas en el teléfono móvil, el tiempo dedicado a la aplicación preferida y el uso de redes sociales como aplicación preferida se relacionan con el hecho de ser mujer. Aunque las correlaciones estimadas son de pequeña magnitud, los factores de Bayes obtenidos sugieren que los datos observados son evidencias muy grandes en favor de la relación entre dichas variables. En los tres casos, los Factores de Bayes estimados en favor de la hipótesis alternativa son mayores que cien, lo que sugiere que, siguiendo la propuesta de Jeffreys (1948), los datos observados son una evidencia decisiva en favor de la idea de correlación genuina entre las variables. Por su parte, no se observan correlaciones de magnitud destacable entre los años de experiencia con el teléfono móvil, el uso de las redes sociales como aplicación preferida y la evitación experiencial. Tampoco se observa correlación destacable entre los años de experiencia y el uso de las redes sociales.

En la Tabla 2 aparecen los resultados de bondad de ajuste de los modelos gráficos estimados con cada uno de los algoritmos utilizados y teniendo en cuenta la partición de los datos en la proporción 70/30. Los algoritmos tabu y $h c$ generan el mismo grafo del mismo modo que los algorit$\operatorname{mos} r \max 2$ y $m m h c$ coinciden en sus estimaciones. Sin embargo, como se aprecia en la Tabla 2, los algoritmos tabuy $h c$ son los algoritmos que mejores índices de bondad de ajuste obtienen cuando los modelos son estimados con el $70 \%$ de los datos. El algoritmo $I A M B$ es el que peores índices de bondad de ajuste muestra. Cuando se utilizan el 30\% de los datos restantes para valorar el sobreajuste de los modelos, se observa que el algoritmo gs es el que obtiene una ligera ventaja. Los algoritmos mmh y rsmax 2 se posicionarían en segundo lugar mientras que el par hc-tabu estarían en tercer lugar. Nuevamente el algoritmo $I A M B$ sería el peor de los algoritmos. No obstante, como se observa en la Figura 1, cuando se procede a la validación cruzada utilizando 2000 
Tabla 1. Coeficientes de correlación adaptados al tipo de variable (Cohen et al., 2003), p-valor clásico del contraste estadístico, intervalo de confianza clásico al 95\% (triángulo inferior izquierdo) y Factor de Bayes favorable a la hipótesis alternativa o FB1o (triángulo superior derecho).

\begin{tabular}{|c|c|c|c|c|c|c|c|c|c|}
\hline & SEX & NA & HT & AT & MUT & TAP & EE & SAS & RS \\
\hline SEX & - & 35377 & 2780 & 0.259 & 4414 & 1246 & 0.165 & 0.067 & $2.34 \times 10^{7}$ \\
\hline NA & $\begin{array}{c}.11 \\
<.001 \\
{[.05, .17]}\end{array}$ & - & 0.327 & $4.24 \times 10^{7}$ & 0.905 & 0.065 & 0.071 & 12510 & 0.041 \\
\hline HT & $\begin{array}{c}-.09 \\
0.003 \\
{[-.14,-.03]}\end{array}$ & $\begin{array}{c}.06 \\
0.036 \\
{[.004, .12]} \\
\end{array}$ & - & 0.039 & 0.294 & $9 \times 10^{99}$ & $6.11 \times 10^{8}$ & $5.97 \times 10^{26}$ & 122162 \\
\hline AT & $\begin{array}{c}-.06 \\
.048 \\
{[-.12,-.001]}\end{array}$ & $\begin{array}{c}.16 \\
<.001 \\
{[.10, .21]}\end{array}$ & $\begin{array}{c}.009 \\
0.747 \\
{[-.05, .07]}\end{array}$ & - & 10132 & 0.038 & 0.037 & 0.210 & 0.037 \\
\hline MUT & $\begin{array}{c}.09 \\
.002 \\
{[.03, .15]}\end{array}$ & $\begin{array}{c}.07 \\
.011 \\
{[.02, .13]}\end{array}$ & $\begin{array}{c}.06 \\
0.041 \\
{[.002, .12]}\end{array}$ & $\begin{array}{c}.10 \\
<.001 \\
{[.04, .16]}\end{array}$ & - & 0.110 & 0.039 & 0.066 & 3436 \\
\hline EE & $\begin{array}{c}-.05 \\
0.083 \\
{[-.11, .01]}\end{array}$ & $\begin{array}{c}-.03 \\
.253 \\
{[-.09, .02]} \\
\end{array}$ & $\begin{array}{c}.20 \\
<.001 \\
{[.14, .25]} \\
\end{array}$ & $\begin{array}{c}.000 \\
.987 \\
{[-.06, .06]} \\
\end{array}$ & $\begin{array}{c}.01 \\
.733 \\
{[-.05, .07]} \\
\end{array}$ & $\begin{array}{c}.24 \\
<.001 \\
{[.18, .29]} \\
\end{array}$ & - & $1.22 \times 10^{31}$ & 0.042 \\
\hline SAS & $\begin{array}{c}-.03 \\
0.276 \\
{[-.09, .03]}\end{array}$ & $\begin{array}{c}.10 \\
<.001 \\
{[.04, .16]}\end{array}$ & $\begin{array}{c}.33 \\
<.001 \\
{[.27, .38]}\end{array}$ & $\begin{array}{c}.06 \\
.062 \\
{[-.003, .11]}\end{array}$ & $\begin{array}{c}.03 \\
.279 \\
{[-.03, .09]}\end{array}$ & $\begin{array}{c}.33 \\
<.001 \\
{[.28, .38]}\end{array}$ & $\begin{array}{c}.35 \\
{[.001} \\
{[.30, .40]}\end{array}$ & - & 403.9 \\
\hline RS & $\begin{array}{c}-.15 \\
<.001 \\
{[-.21,-.09]}\end{array}$ & $\begin{array}{c}-.01 \\
.641 \\
{[-.07, .04]}\end{array}$ & $\begin{array}{c}.12 \\
<.001 \\
{[.06, .17]}\end{array}$ & $\begin{array}{c}.004 \\
.891 \\
{[-.05, .06]}\end{array}$ & $\begin{array}{c}-.09 \\
.003 \\
{[-.15,-.03]}\end{array}$ & $\begin{array}{c}.14 \\
<.001 \\
{[.08, .20]}\end{array}$ & $\begin{array}{c}-.02 \\
.604 \\
{[-.07, .04]}\end{array}$ & $\begin{array}{c}.13 \\
<.001 \\
{[.07, .18]}\end{array}$ & - \\
\hline
\end{tabular}

Nota. SEX: sexo ( 1 = hombre, 0 = mujer), NA: número de aplicaciones instaladas en el teléfono móvil, HT: horas diarias invertidas en el teléfono móvil, AT: años de experiencia utilizando teléfono móvil, MUT: tener más de un teléfono móvil ( 0 = no, 1= sî), TAP: tiempo diario invertido en la aplicación preferida, EE: puntuación en la escala AAQ-II de evitación experiencial, SAS: puntuación en la escala SAS-SV de adicción al móvil, y RS: considerar las redes sociales como la aplicación preferida $(0=$ no, $1=$ sí). Todos los contrastes son bilaterales.

Tabla 2. Índices de bondad de ajuste para cada uno de los algoritmos utilizando el $70 \%$ de los datos para la estimación y el $30 \%$ restante para la valoración del sobreajuste.

\begin{tabular}{|c|c|c|c|c|c|c|}
\hline Algoritmo & TABU & $\mathrm{HC}$ & RSMAX2 & MMHC & GS & IAMB \\
\hline FRP & 1.44 & 1.44 & 1.1 & 1.1 & 1.22 & 0.78 \\
\hline Número de tests & 288 & 140 & 245 & 220 & 212 & 292 \\
\hline Nodos & 9 & 9 & 9 & 9 & 9 & 9 \\
\hline Arcos & 13 & 13 & 10 & 10 & 11 & 7 \\
\hline Parámetros & 22 & 22 & 19 & 19 & 20 & 16 \\
\hline LL-70 & -15843.82 & -15843.82 & $-15855 \cdot 34$ & -15855.34 & -15862.58 & -16340.11 \\
\hline AIC-70 & -15865.82 & -15865.82 & $-15874 \cdot 34$ & $-15874 \cdot 34$ & -15882.58 & -16356.11 \\
\hline BIC-70 & -15917.5 & -15917.5 & -15918.98 & -15918.98 & $-15929 \cdot 56$ & -16393.7 \\
\hline LL-30 & -6911.77 & -6911.77 & -6914.6 & -6914.6 & -6917.08 & -7085.75 \\
\hline AIC-30 & -6933.77 & -6933.77 & -6933.6 & -6933.6 & -6937.08 & -7101.75 \\
\hline $\mathrm{BIC}-30$ & -6985.46 & -6985.46 & -6978.23 & -6978.23 & -6975.6 & -7132.57 \\
\hline
\end{tabular}

Nota. FRP: factor de ramificación promedio, TABU: algoritmo tabu search, HC: algoritmo hill-climbing, RSMAX2: algoritmo restricted maximization, MMHC: algoritmo max-min hill-climbing, GS: algoritmo grow-shrink, IAMB: algoritmo incremental association, LL: logaritmo de la verosimilitud, AIC: Criterio de Información Akaike, y BIC: Criterio de Información Bayesiano.

particiones aleatorias de los datos, se observa que los mejores algoritmos para estimar la estructura de dependencia contenida en los datos son los algoritmos hc y tabu.
En la Figura 2 aparece representado el modelo gráfico estimado con los algoritmos hc y tabu y que puede considerase, por tanto, como el más aceptable. Como se puede 


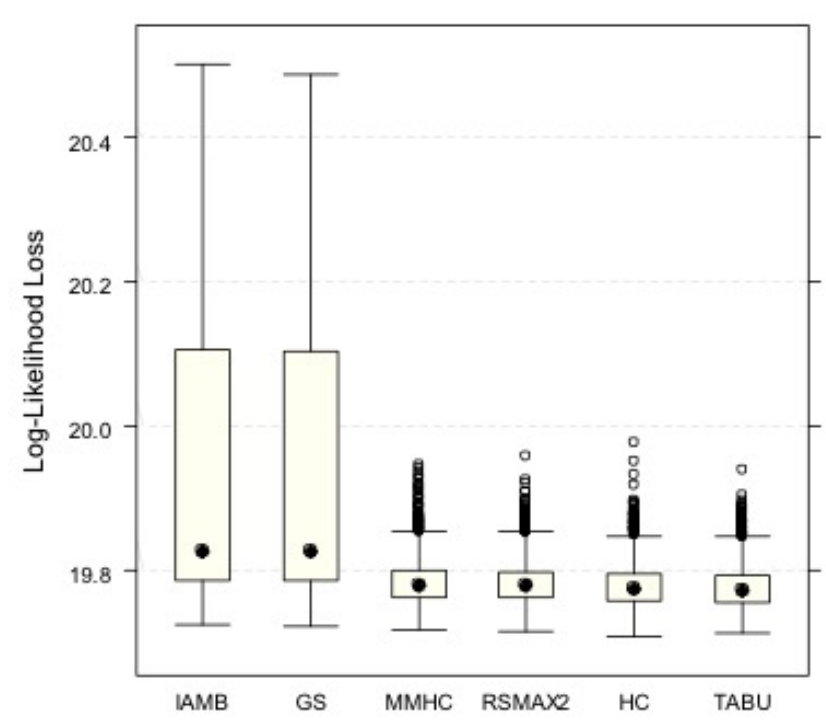

Figura 1. Resultados de los análisis de validación cruzada. Nota. Pérdida en el logaritmo de la verosimilitud para cada una de las 2000 particiones realizadas en la base de datos. Los gráficos de caja están ordenados en orden decreciente atendiendo a la mediana.

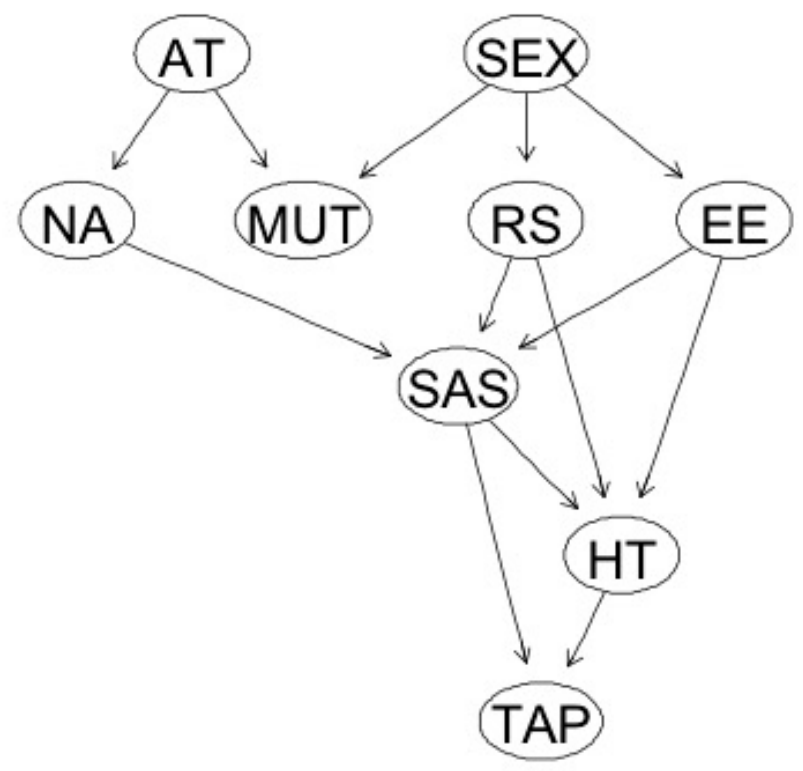

Figura 2. Modelo de red bayesiana estimado con los algoritmos tabu y hc.

Nota. AT: años de experiencia con el teléfono móvil, SEX: sexo, NA: número de aplicaciones instaladas en el teléfono móvil, MUT: tener más de un teléfono móvil, RS: considerar las redes sociales como la aplicación preferida, EE: puntuación en la escala AAQ-II de evitación experiencial, SAS: puntuación en la escala SAS-SV de adicción al móvil, HT: horas diarias invertidas en el teléfono móvil, y TAP: tiempo diario invertido en la aplicación preferida.

apreciar, las variables sexo y años de experiencia con el teléfono móvil son las únicas que no dependen de cualquier otra variable. Por su parte, la puntuación en la escala de adicción al móvil SAS-SV depende de la evitación experiencial, de considerar las aplicaciones de redes sociales como las aplicaciones preferidas y del número de aplicaciones instaladas en el dispositivo móvil. Por otro lado, el grafo estimado también muestra que el número de horas invertidas en el teléfono móvil depende de los niveles de evitación ex- periencial, de la preferencia por el uso de las redes sociales y de la puntuación en la escala de adicción al móvil.

Para poder valorar el papel que tienen cada una de las aristas del grafo, se ha estimado el impacto que tiene la eliminación de cada una de ellas. En la Tabla 3 aparece la reducción que se produce en los principales índices de bondad de ajuste del nuevo modelo cuando un enlace particular es eliminado del grafo estimado (Figura 2). Cuanto mayor es la reducción que se produce en el BIC y estadísticos relacionados al eliminar un enlace, más relevante puede considerarse dicho enlace en el modelo obtenido. Así, el enlace más relevante del modelo presentado en la Figura 2, y el que más empeora todos los índices de bondad de ajuste si es eliminado del modelo, es el que va desde las horas diarias de uso del teléfono móvil hasta el tiempo diario invertido en la aplicación preferida (ver Tabla 3). Por su parte, el segundo enlace más fuerte que se identifica en el modelo es el que va desde la evitación experiencial hasta la puntuación en la escala de adicción al móvil. Estos dos enlaces junto al que va de la puntuación en la escala SAS-SV hasta el número de horas invertidas en el teléfono móvil serían los arcos más relevantes del modelo. Si estos enlaces fuesen eliminados del modelo los índices de bondad de ajuste empeorarían. Dicho de otro modo, estas serían las relaciones que se establecen con más fuerza entre las variables incluidas en el modelo. Por su parte, el enlace que va desde el sexo a la evitación experiencial es el menos fuerte y podría eliminarse del modelo sin que se observasen repercusiones drásticas en los índices de bondad de ajuste estimados.

Tabla 3. Cambio que se produce en la bondad de ajuste del modelo cuando se elimina un enlace dirigido de la red bayesiana.

\begin{tabular}{ccccc}
\hline Desde & Hasta & LL & AIC & BIC \\
\hline HT & TAP & -587.06 & -586.06 & -583.71 \\
EE & SAS & -80 & -79 & -76.66 \\
SAS & HT & -42.72 & -41.72 & -39.37 \\
SEX & RS & -13.41 & -12.41 & -10.06 \\
AT & NA & -14.01 & -13.01 & -10.66 \\
RS & SAS & -12.02 & -11.02 & -8.67 \\
NA & SAS & -8.79 & -7.79 & -5.44 \\
SAS & TAP & -7.77 & -6.77 & -4.42 \\
SEX & MUT & -5.5 & -4.5 & -2.15 \\
EE & HT & -6.17 & -5.17 & -2.82 \\
RS & HT & -4.58 & -3.58 & -1.23 \\
AT & MUT & -6.33 & -5.33 & -2.99 \\
SEX & EE & -1.51 & -0.51 & 1.84 \\
\hline
\end{tabular}

Nota. SEX: sexo, NA: número de aplicaciones instaladas en el teléfono móvil, HT: horas diarias invertidas en el teléfono móvil, AT: años de experiencia utilizando teléfono móvil, MUT: tener más de un teléfono móvil, TAP: tiempo diario invertido en la aplicación preferida, EE: puntuación en la escala AAQ-II de evitación experiencial, SAS: puntuación en la escala SAS-SV de adicción al móvil, RS: considerar las redes sociales como la aplicación preferida, LL: logaritmo de la verosimilitud, AIC: Criterio de Información Akaike, y BIC: Criterio de Información Bayesiano. 


\section{Discusión}

Este estudio tuvo por objeto investigar la relación que existe entre diversas variables que pueden vincularse con la adicción al móvil o el uso problemático del mismo y el de las redes sociales. La primera idea que se planteaba para ser testada era la existencia de una relación entre la variable evitación experiencial y el uso abusivo del móvil. Los resultados muestran, tanto si analizamos las correlaciones obtenidas entre las mismas, como en la red bayesiana obtenida, que efectivamente existe una relación entre las variables. Este resultado nos plantea la posibilidad de que el teléfono móvil es usado como vía de escape a las emociones y pensamientos negativos internos. Estos resultados están en consonancia con algunos de los trabajos recientes desarrollados por Chóliz et al. (2009) y Carbonell et al. (2012) cuando señalan que las mujeres lo utilizan para enfrentarse a estados de ánimo displacenteros o para aliviar el malestar emocional, y tal como señalan García-Oliva y Piqueras (2016). En este sentido, si el patrón de uso del teléfono móvil está condicionado por la evitación de sensaciones negativas internas, podría desembocar en problemas a largo plazo. La relación direccional observada entre la evitación experiencial y la adicción al móvil sugiere que la segunda depende de la primera como parece suceder en otros trastornos adictivos (p.e., Buckner y Zvolensky, 2014; Garey et al. 2016; Hayes et al. 1996; Levin et al., 2016; Watson et al., 2017). No obstante, y al tratarse de un estudio correlacional-exploratorio, estas relaciones de dependencia deben interpretarse con cautela y estudiarse bajo otras metodologías de investigación que permitan aproximarnos con más precisión a explicaciones causales. En cualquier caso, nuestros datos sugieren que un uso no adaptativo del móvil está relacionado con la evitación experiencial y, por tanto, sería deseable prestar atención a este hecho tanto desde el punto de vista clínico como científico.

Respecto a la relación que se esperaba encontrar entre los niveles de evitación experiencial y el uso de las redes sociales, no se observa la existencia de una relación directa entre dichas variables. Hay que tener en cuenta, no obstante, que la adicción al móvil es una variable de convergencia (un efecto común potencial) respecto al uso de las redes sociales y de la evitación experiencial. Por consiguiente, teniendo en cuenta el formalismo de las redes bayesianas, cuando el nivel de adicción al móvil es conocido, la evitación experiencial y las redes sociales se tornan condicionalmente dependientes. En cualquier caso, el modelo de red bayesiana estimado sugiere que la relación que se observa entre estas variables estaría mediada por el sexo. En este sentido, como se predecía y como plantean, por ejemplo, Chóliz et al. (2009), Gil et al. (2015) y Tresáncoras et al. (2017), existe una relación entre el sexo y un uso inapropiado del móvil. Sin embargo, según los resultados que se han obtenido, esta relación estaría también condicionada por el uso de las redes sociales (Figura 2). En este estudio, al igual que en los estudios precedentes, se ha observado que las mujeres utilizan el smartphone más que los hombres en términos de tiempo dedicado a las redes sociales que, por otro lado, suele ser su aplicación más preferida. Si lo revisamos desde el punto de vista gráfico, vemos que la relación entre sexo y horas de uso del teléfono móvil está mediada por considerar como aplicación preferida las redes sociales (Figura 2).

Desde un punto de vista teórico, nuestros resultados son consistentes con la Teoría de Monitorización Hipernatural de la Adicción al smartphone (Veissière y Stendel, 2018). Esta teoría sostiene que no existe algo intrínsecamente adictivo en el teléfono móvil. Más bien, Veissière y Stendel (2018) sugieren que la adicción al móvil es consecuencia de la expectativa social en términos de las recompensas que se obtienen al conectar con otras personas. Este componente social podría explicar tanto el inicio como el mantenimiento de la adicción al móvil, así como la dimensión neurofisiológica que se observa en adicciones a sustancias y en otras adicciones comportamentales (p.e., Bohbot, Del Balso, Conrad, Konishi y Leyton, 2013; Sussman, Harper, Stahl y Weigle, 2018). Nuestros resultados suponen un apoyo a esta teoría dado que los niveles de adicción al móvil pueden ser explicados en función, al menos en parte, de la interacción con las redes sociales y como consecuencia de la evitación sistemática de experiencias internas desagradables para la persona. De todos modos, pese a que esta teoría requiere ser contrastada empíricamente, sobre todo en lo que se refiere a los correlatos neurofisiológicos, nuestros resultados son consistentes con sus postulados.

Las adicciones comportamentales no están recogidas en el DSM-5 (APA, 2013), exceptuando la adicción al juego. No obstante, se observa que a pesar de las consecuencias positivas que pueden derivarse del uso de las tecnologías de la información, por ejemplo, del smartphone, también existen consecuencias negativas de un uso exacerbado o no funcional del mismo. En este sentido, Potenza, Higuchi y Brand (2018) abogan por la continuidad en el estudio de las adicciones comportamentales con el fin de mejorar las estrategias de intervención. Y no solo centrar la atención en el juego patológico, sino a otro tipo de conductas que pueden desembocar en adicciones. Tal como se ha observado en este trabajo, parece ser que la evitación experiencial tiene algún papel con relación al uso abusivo del móvil. En este sentido, sugerimos que se investigue si las intervenciones en este tipo de problemas deberían ir orientadas a favorecer un mayor contacto con uno mismo, $y$, como recogen todas aquellas intervenciones que toman de base el mindfulness, prestar una mayor atención a los estados internos independientemente de que sean positivos o negativos. Si, como se deduce a partir de nuestros resultados, algunas personas utilizan el móvil para huir o evitar sentimientos negativos buscando cierto tipo de alivio inmediato, a la larga podrían acaecer consecuencias nega- 
tivas para el individuo desembocando, presumiblemente, en adicción comportamental.

Una de las limitaciones de nuestro trabajo es que se trata de un estudio correlacional y exploratorio (Nosek, Ebersole, DeHaven y Mellor, 2018). Aunque este tipo de estudios son útiles, serían necesarios estudios longitudinales, e incluso experimentales, para poder analizar realmente el impacto de unas variables sobre otras. A pesar de que los resultados sugieren la existencia de una relación entre la evitación experiencial y el uso desadaptativo del móvil, podría ser interesante llevar a cabo estudios con personas con diagnóstico clínico de este trastorno para ser comparadas con población general y poder observar los patrones comportamentales en ambos grupos. Otra de las limitaciones del estudio tiene que ver con el procedimiento de recogida de datos. Pese a permitir el acceso a un amplio espectro de participantes, no se pueden controlar ciertas variables, como por ejemplo la deseabilidad social en las respuestas al cuestionario. Por otra parte, no se ha realizado un estudio segmentado por edades, en donde pudiesen ser observadas diferencias entre grupos o distintas etapas vitales. Hay que tener en cuenta que el rango de edad de los participantes estudiados es muy grande y esa dispersión podría haber afectado a los resultados obtenidos en algún sentido. En este sentido, futuros trabajos podrían incidir en estos aspectos, dado que, como sugiere Odgers (2018), las consecuencias del uso de las tecnologías no son iguales dependiendo de la persona de que se trate o en función del estadio evolutivo en el que se encuentre.

Creemos que una de las líneas futuras de investigación debería indagar realmente en si se pueden catalogar o no como adictivas, aquellas conductas inapropiadas de uso de las tecnologías de la información. Como señalan Potenza et al. (2018), la comprensión de los procesos biológicos, psicológicos y sociales que están a la base de las adicciones comportamentales puede mejorar tanto las estrategias de prevención y tratamiento de las mismas. En cualquier caso, deberíamos abogar por un buen uso de los teléfonos móviles, o la tecnología en general, para hacer más fructífera nuestra vida en sociedad. No es cuestión de prohibir o rechazar las tecnologías porque se usen mal, sino que más bien, como sugiere Abelson (1997) en el contexto de los análisis de datos estadísticos, educar sobre su buen uso.

\section{Conflicto de intereses}

Los autores no tienen conflictos de intereses que declarar.

\section{Referencias}

Abelson, R. P. (1997). On the surprising longevity of flogged horses: why there is a case for the significance test. Psychological Science, 8, 12-15. doi:10.1111/j.1467-9280.1997. tb00536.x.
American Psychiatric Association (1994). Diagnostic and Statistical Manual of Mental Disorders (DSM-IV). Washington, DC: American Psychiatric Association.

American Psychiatric Association (2000). Diagnostic and Statistical Manual of Mental Disorders (DSM-IV-TR). Barcelona: Masson.

American Psychiatric Association (2013). Diagnostic and Statistical Manual of Mental Disorders (DSM-5). Barcelona: Panamericana.

Arab, E. y Díaz, A. (2015). Impacto de las redes sociales e internet en la adolescencia: aspectos positivos y negativos. Revista Médica Clínica Las Condes, 26, 7-13.

Babín, F. A. (2009). Estudio del uso problemático de las tecnologías de la información, la comunicación y el juego entre los adolescentes y jóvenes de la ciudad de Madrid. Trastornos Adictivos, 11, 151-163. doi:10.1016/ S1575-0973(09)72407-9.

Bahrami, S. y Asghari, F. (2017). A controlled trial of acceptance and commitment therapy for addiction severity in methamphetamine users: Preliminary study. Archives of Psychiatry and Psychotherapy, 19, 49-55. doi:10.12740/ APP $/ 68159$.

Bohbot, V. D., Del Balso, D., Conrad, K., Konishi, K. y Leyton, M. (2013). Caudate nucleus-dependent navigational strategies are associated with increased use of addictive drugs. Hippocampus, 23, 973-984. doi:10.1002/ hipo. 22187.

Bond, F. W., Hayes, S. C., Baer, R. A., Carpenter, K. M., Guenole, N., Orcutt, H. K., ... Zettle, R. D. (2011). Preliminary Psychometric Properties of the acceptance and action questionnaire-II: a revised measure of psychological inflexibility and experiential avoidance. Behaviour Therapy, 42, 676-688.

Bragazzi, N. L. y Puente, G. (2014). A proposal for including nomophobia in the new DSM-5. Psychology Research and Behavior Management, 7, 155-160.

Buchinger, S., Kriglstein, S., Brandt, S. y Hlavacs, H. (2011). A survey on user studies and technical aspects of mobile multimedia applications. Entertainment Computing, 2, 175-190. doi:10.1016/j.entcom.2011.02.001.

Buckner, J. D. y Zvolensky, M. J. (2014). Social anxiety and coping motives for cannabis use: the impact of experiential avoidance. Psychology of Addictive Behaviors, 28, 568574.

Capon, H., Hall, W., Fry, C. y Carter, A. (2016). Realising the technological promise of smartphone in addiction research and treatment: an ethicall review. International Journal of Drug Policy, 36, 47-57.

Carbonell, X., Fúster, H., Chamarro, A. y Oberst, U. (2012). Adicción a internet y móvil: una revisión de estudios empíricos españoles. Papeles del Psicólogo, 33, 83-89.

Chóliz, M. (2012). Mobile-phone addiction in adolescence: the test of mobile phone dependence (TMD). Progress in Health Sciences, 2, 33-44. 
Chóliz, M., Villanueva, V. y Chóliz, M. C. (2009). Ellos, ellas y su móvil: uso, abuso (¿y dependencia?) del teléfono móvil en la adolescencia. Revista Española de Drogodependencias, 34, 74-88.

Cohen, J., Cohen, P., West, S. G. y Aiken, L. S. (2003). Applied multiple regression/correlation analysis for the behavioral sciences ( $\left.3^{\mathrm{a}} \mathrm{ed}\right)$. Mahwah, NJ: Laurence Erlbaum.

Cowell, R. G., Dawid, A. P., Lauritzen, S. L. y Spiegelhalter, D. J. (1999). Probabilistic networks and expert systems. Harrisonburg, VA: Springer.

De-Sola, J., Rodríguez, F. y Rubio, G. (2016). Cell-phone addiction: A review. Frontiers in Psychiatry, 7. doi: 10.3389/ fpsyt.2016.00175.

Deursen, A. J., Bolle, C. L., Hegner, S. M. y Kommers, P. A. (2015). Modeling habitual and addictive smartphone behavior: the role of smartphone usage, types, emotional intelligence, social stress, self-regulation, age and gender. Computers in Human in Behavior, 45, 411-420. doi:10.1016/j.chb.2014.12.039.

Echeburúa, E. y Corral, P. (2010). Adicción a las nuevas tecnologías y a las redes sociales en jóvenes: un nuevo reto. Adicciones, 22, 91-96.

Edwards, W. (1998). Hailfinder. Tools for and experiences with Bayesian normative modeling. American Psychologist, 53, 416-428.

García-Oliva, C. y Piqueras, J. A. (2016). Experiential avoidance and technological addictions in adolescents. Journal of Behavioral Addictions, 5, 293-303. doi:10.1556/2006.5.2016.041.

Garey, L., Farris, S. G., Schmidt, N. B. y Zvolensky, M. J. (2016). The role of smoking-specific experiential avoidance in the relation between perceived stress and tobacco dependence, perceived barriers to cessation, and problems during quit attempts among treatment-seeking smokers. Journal of Contextual Behavioral Science, 5, 58-63.

Gil, F., del Valle, G., Oberst, U. y Chamarro, A. (2015). Nuevas tecnologías - ¿Nuevas patologías? El smartphone y el fear of missing out. Aloma. Revista de Psicologia, Ciències de l'Educació $i$ de l'Esport, 33, 77-83

Griffiths, M. (2000). Does Internet and computer "addiction" exist? Some case study evidence. Cyberpsychology $\mathcal{E}^{\circ}$ Behavior, 3, 211-218.

Gustafson, D. H., McTavish, F. M., Chih, M. I., Atwood, A. K., Johnson, R. A., Boyle, M. G., ...Shah, D. (2014). A smartphone application to support recovery from alcoholism a randomized clinical trial. JAMA Psychiatry, 71, 566-572.

Hardell, L., Carlberg, M. y Hansson, K. L. (2011). Pooled analysis of case-control studies malignant brain tumors and the use of mobile and cordless phones including living and deceased subjects. International Journal of Oncology, 38, 1465-1474. doi:10.3892/ijo.2011.947.

Hayes, S. C., Bissett, R. T., Strosahl, K., Wilson, K., Pistorello, J., Toamino, D., ...Follete, W. C. (2000). Psicometric properties of the acceptance and action questionnaire (AAQ). Manuscrito inédito, Departamento de Psicología, Universidad de Nevada, Reno.

Hayes, S. C., Brownstein, A. S., Zettle, R. D., Rosenfard, I. y Korn, Z. (1986). Rule-governed behavior and sensitivity to changing consequences of responding. Journal of the Experimental Analysis of Behavior, 45, 237-256.

Hayes, S. C., Strosahl, K. D. y Wilson, K. G. (1999). Acceptance and commitment therapy. New York: The Guilford Press.

Hayes, S. C., Strosahl, K., Wilson, K. G., Bissett, R. T., Pistorello, J., Toarmino, D., ... McCurry, S. M. (2004). Measuring experiential avoidance: a preliminary test of a working model. The Psychological Record, 54, 553-578.

Hayes, S. C., Wilson, K. G., Gifford, E. V., Follete, V. M. y Stroashal, K. (1996). Experiential avoidance and disorder: a functional dimensional approach to diagnosis and treatment. Journal of Consulting and Clinical Psychology, 64, 1152-1168.

Hayes, S. C., Zettle, R. D. y Rosenfarb, I. (1989). Rule following. En S. C. Hayes (Ed.), Rule-governed behavior cognition, contingencies and instructional control (pp. 191-220). New York: Plenum Press.

JASP Team (2018). JASP (Version 0.8.5) [Computer software].

Jeffreys, H. (1948). Theory of probability ( $2^{\mathrm{a}}$ Ed.). Oxford: Oxford University Press.

Jeon, M. y De Boeck, P. (2017). Decision qualities of Bayes Factor and $\mathrm{p}$ value-based hypothesis testing. Psychological Methods, 22, 340-360. doi: 10.1037/met0000140.

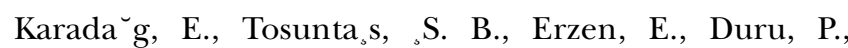

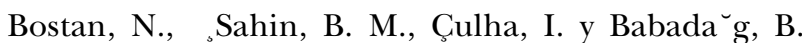
(2015). Determinants of phubbing, which is the sum of many virtual addictions: A structural equation model. Journal of Behavioral Addictions, 4, 60-74. doi:10.1556/2006.4.2015.005.

Kass, R. E. y Raftery, A. E. (1995). Bayes factors. Journal of the American Statistical Association, 90, 773-795. doi: 10.1080/01621459.1995.10476572.

Klawer, S. G., Guo, F., Simons-Morton, B. C., Ouimet, M. C., Lee, S. E. y Dingus, T. A. (2014). Distracted driving and risk of road crashes among novice and experienced drivers. The New England Journal of Medicine, 370, 54-59 doi:10.1056/NEJMsa1204142.

Koller, D. y Friedman, N. (2010). Probabilistic graphical models. Principles and techniques. Cambridge, MA: MIT Press.

Krasnova, H., Abramova, O., Notter, I. y Baumann, A. (2016). Why phubbing is toxic for your relationship: Understanding the role of smartphone jealousy among "Generation $Y$ ” users. Comunicación presentada en el Twenty-Fourth European Conference on Information Systems (ECIS), Estambul, Turquía.

Kuhn, E., Kanuri, N., Hoffman, J. E., Garvert, D. W., Ruzek, J. L. y Tailor, C. B. (2017). A randomized controlled trial of a smartphone app for postraumatic stress disorders 
symptoms. Journal of Consulting and Clinical Psychology, 85, 267-273. doi:10.1037/ccp0000163.

Kwon, M., Kim, D-J, Cho, H. y Yang, S. (2013b). The Smartphone Addiction Scale: Development and Validation of Short Version for Adolescents. PLoS One 8, e83558. doi:10.1371/journal.pone.0083558.

Kwon, M., Lee, J-Y., Won, W-Y., Park, J-W., Min, J-A., Hahn, C., ... Kim, D. J. (2013a). Development and Validation of a Smartphone Addiction Scale (SAS). PLoS One, 8, e56936. doi:10.1371/journal.pone.0056936.

Lee, S., Kang, H. y Shin, G. (2015). Head flexion angle while using a smartphone. Ergonomis 58, 220-226. doi:10 $.1080 / 00140139.2014 .967311$.

Levin, M. E., Lillis, J., Seeley, J., Hayes, S. L., Pistorello, J. y Biglan, A. (2016). Exploring the relationship between experiential avoidance, alcohol use disorders, and alcohol-related problems among first-year college students. Journal of American College Health, 60, 443-448.

López, J., García, J., De la Fuente, L. y De la Fuente, E. I. (2007). Las redes bayesianas como herramientas de modelado en psicología. Anales de Psicología, 23, 577-581.

López-Fernández, O. (2015). Short version of the Smartphone addiction scale adapted to Spanish and French: Towards a cross-cultural research in problematic mobile phone use. Addictive Behaviors, 64, 275-280. doi:10.1016/j.addbeh.2015.11.013.

Marín, M., Carballo, J. L. y Coloma-Carmona, A. (2018). Rendimiento académico y cognitivo en el uso problemático de Internet. Adicciones, 30, 101-110. doi:10.20882/ adicciones.844.

McDaniel, B. T. y Coyne, S. M. (2016). "Technoference": The interference of technology in couple relationships and implications for women's personal and relational well-being. Psychology of Popular Media Culture, 5, 85-98. doi:10.1037/ppm0000065.

Mendoza, M. I. y Cuñarro, L. (2016). Celular e intersubjetividad. Omnia, 22, 20-31.

Nagarajan, R., Scutari M. y Lèbre, S. (2013). Bayesian networks in $R$ with applications in systems biology. Nueva York: Springer.

Nosek, B., Ebersole, C., DeHaven, A. y Mellor, D. (2018). The preregistration revolution. Proceedings of the National Academy of Sciences, 115, 2600-2606. doi:10.1073/ pnas. 1708274114.

Odgers, C. (2018). Smartphone are bad for some teens, not all. Nature, 554, 432-434. doi:10.1038/d41586-01802109-8.

Orozco, G. O. (2015). Televisión y producción de significados (tres ensayos). Revista Comunicación y Sociedad, 1, 20-36.

Pedrero, E. J., Rodríguez, M. T. y Ruiz, J. M. (2012). Adicción o abuso del teléfono móvil. Revisión de la literatura. Adicciones, 24, 132-152.
Pedrero-Pérez, E. J., Ruiz-Sánchez de León, J. M., Rojo-Mota, G., Llanero-Luque, M., Pedrero-Aguilar, J., Morales-Alonso, S. y Puerta-García, C. (2018). Tecnologías de la Información y la Comunicación (TIC): uso problemático de internet, videojuegos, teléfonos móviles, mensajería instantánea y redes sociales mediante el MULTICAGE-TIC. Adicciones, 30, 19-32. doi:10.20882/adicciones.806.

Potenza, M. N., Higuchi, S. y Brand, M. (2018). Broaden behavioural addiction research. Nature, 555, 30. doi: 10.1038/d41586-018-02568-z.

Puga, J. L., Krzywinski, M. y Altman, N. (2015). Bayesian networks. Nature Methods, 12, 799-800. doi:10.1038/ nmeth.3550.

Roberts, J. A. y David, M. E. (2016). My life has become a major distraction from my cell phone: Partner phubbing and relationship satisfaction among romantic partners. Computers in Human Behavior, 54, 134-141. doi:10.1016/j. chb.2015.07.058.

Rodríguez, A. (2015). Hábitos y problemas del sueño en la infancia y adolescencia en relación al patrón de uso del teléfono móvil: estudio transversal. Tesis doctoral. Departamento de Pediatría, Obstetricia y Ginecología, Universidad de Valencia. Recuperado de http://hdl.handle. net/10550/50055.

Rouder, J. N., Speckman, P., Sun, D. y Morey, R. (2009). Bayesian t tests for accepting and rejecting the null hypothesis. Psychonomic Bulletin E Review, 16, 225-237.

Ruiz, F. J., Langer, A. I., Luciano, C., Cangas, A. J. y Beltrán, I. (2013). Measuring experiential avoidance and psychological inflexibility: The Spanish version of the Acceptance and Action Questionnaire - II. Psicothema, 25, 123-129. doi:10.7334/psicothema2011.239.

Ruiz-Ruano, A. M. (2015). Aprendizaje estructural de redes bayesianas para modelar el emprendimiento académico de base sostenible y tecnológica. Tesis doctoral no publicada, Facultad de Ciencias de la Salud, Universidad Católica San Antonio de Murcia. Recuperado de http://hdl.handle. net/10952/1556.

Scutari, M. (2010). Learning Bayesian Networks with the bnlearn R package. Journal of Statistical Software, 35, 1-22. doi:10.18637/jss.v035.i03.

Scutari, M. y Denis, J. B. (2014). Bayesian networks: with examples in R. Boca Raton, FL: CRC Press.

Seoane, A. y Álvarez, G. (2012). Nuevas tecnologías aplicadas al tratamiento del tabaquismo: Doctor Fum. Revista Española de Drogodependencias, 37, 319-332.

Simó, C., Martínez, A., Ballester, M. L. y Domínguez, A. (2017). Instrumentos de evaluación del uso problemático del teléfono móvil/smartphone. Health and Addictions, 17, 5-14.

Sussman, C. J., Harper, J. M., Stahl, J. L. y Weigle, P. (2018). Internet and video game addictions. Diagnosis, epidemiology, and neurobiology. Child and Adolescent Psychia- 
tric Clinics of North America, 27, 307-326. doi:10.1016/j. chc.2017.11.015

Tresáncoras, A. G., García-Oliva, C. y Piqueras, J. A. (2017). Relación del uso problemático del Whatsapp con la personalidad y la ansiedad en adolescentes. Health and Addictions, 17, 27-36.

Veissière, S. P. y Stendel, M. (2018). Hypernatural monitoring: A social rehearsal account of smartphone addiction. Frontiers in Psychology, 9, 1118. doi:10.3389/ fpsyg.2018.00141.

Wang, X., Xie, X., Wang, Y., Wang, P. y Lei, L. (2017). Partner phubbing and depression among married Chinese adults: The roles of relationship satisfaction and relationship length. Personality and Individual Diferences, 110, 12-17. doi:10.1016/j.paid.2017.01.014.

Watson, N., Heffner, J., McClure, J. y Bricker, J. (2017). Relationships Between Social Anxiety and Smoking-Specific Experiential Avoidance. Journal of Dual Diagnosis, 13, 1-5. doi: 10.1080/15504263.2016.1248310.Wilson, K. G. y Luciano, M. C. (2012). Terapia de Aceptación y Compromiso (ACT). Un tratamiento conductual orientado a los valores. Madrid, España: Pirámide.

Wulfert, E., Greenway, D. E., Farkas, P., Hayes, S. C. y Dougher, M. J. (1994). Correlation between self-reported rigidity and rule-governed insensitivity to operant contingencies. Journal of Applied Behavior Analysis, 27, 659-671.

Zarghami, M., Khalilian, A., Setareh, J. y Salehpour, G. (2015). The impact of using cell phones after light-out on sleep quality, headache, tiredness, and distractibility among students of a university in North of Iran. Iranian Journal of Psychiatry and Behavioral Sciences, 9, e2010. doi:10.17795/ijpbs.2010.

Zhitomirsky-Geffet, M. y Blau, M. (2016). Cross generational analysis of predictive factors of addictive behavior in smartphone usage. Computers in Human Behavior, 64, 682-693. 